\title{
The value of mid-trimester fetal liver length measurement in prediction of gestational diabetes in Iraqi women
}

\author{
H A K Showman, ${ }^{1} \mathrm{MB} \mathrm{ChB} ; \mathrm{H} \mathrm{A} \mathrm{G} \mathrm{Al-Rawi,}{ }^{1} \mathrm{MB} \mathrm{ChB} ; \mathbf{M}$ A G Zghair ${ }^{2} \mathrm{MB} \mathrm{ChB}$ \\ ${ }^{1}$ Department of Obstetrics and Gynecology, College of Medicine, Al-Mustansiriya University, Iraq \\ ${ }^{2}$ Department of Radiology, College of Medicine, Al-Mustansiriya University, Iraq
}

Corresponding author: H A K Showman (hind_showman@yahoo.com)

Background. Gestational diabetes (GD) is a hyperglycaemic state specific to pregnancy. A well-tolerated, quick and minimally-invasive method for the early detection of GD in pregnancy is lacking.

Objective. To evaluate the correlation between the mid-trimester length of the fetal liver and GD.

Methods. A prospective study was conducted at the National Endocrine and Diabetic Center, College of Medicine, Al-Mustansiriya University, Iraq and the Obstetric and Gynecological Department at Al-Yarmouk Teaching Hospital, Iraq, from September 2016 to September 2018. One-hundred-and-twenty singleton pregnant women at high risk for gestational diabetes mellitus (GDM) were recruited. An ultrasound scan was performed at 23 weeks' gestation followed by a $75 \mathrm{~g}$ oral glucose tolerance test (OGTT) at 24 weeks. A correlation was assessed between the mid-trimester fetal liver length (FLL) measurement and the OGTT.

Results. Mean (SD) FLL values were significantly higher among GDM patients v. healthy pregnant females respectively: 37.2 (3.4) v. 33.1 (2.7); $p<0.001$. Univariate analysis showed that FLL in GDM group was 1.6 times the non-GDM group (odds ratio (OR) 1.6; 95\% confidence interval (CI) 1.31 - 1.96). There was no significant change after adjustment for parity and cumulative risk factors. Based on the above, FLL is an independent predictor for GDM (OR 1.6; 95\% CI $1.30-1.96 ; p<0.05$ ).

Conclusion. FLL measurement by ultrasound at 23 weeks' gestation is a feasible alternative to OGGT for the early detection of GDM in high-risk patients.

S Afr J Obstet Gynaecol 2019;25(3):100-102. https://doi.org/10.7196/SAJOG.2019.v25i3.1439

Gestational diabetes mellitus (GDM) has been defined as 'hyperglycaemia first recognised during pregnancy' and has more recently been described by the American Diabetes Association (ADA) as 'diabetes diagnosed in the second or third trimester of pregnancy that is not clearly overt diabetes ${ }^{[1]}$ with an incidence between $10-15 \%$ of pregnancies, depending on the diagnostic criteria used. ${ }^{[2]}$ The prevalence of GDM is expected to increase as the epidemic of obesity continues. ${ }^{[3]}$ Risk factors for gestational diabetes include glycosuria in the first trimester, glycosuria on 2 or more occasions in the second or third trimester, ${ }^{[3]}$ polyhydramnios in the current pregnancy, ${ }^{[5]}$ macrosomia in the current pregnancy, ${ }^{[6]}$ previous unexplained stillbirth ${ }^{[7]}$ obesity $(\mathrm{BMI}>30){ }_{{ }^{[8]}}$ a previous baby $>4.5 \mathrm{~kg},{ }^{[9]}$ previous congenital abnormality, ${ }^{[10]}$ and age $>35$ years. ${ }^{[1]}$ Other risk factors include previous GDM, ${ }^{[12]}$ history of diabetes in a first-degree relative, ${ }^{[13]}$ and belonging to ethnicities with a high prevalence of diabetes, e.g. South Asian, black Caribbean and Middle Eastern. ${ }^{[4]}$ GDM assessment in the first trimester is still poor, resulting in the delay of GDM diagnosis to the late second or early third trimester. ${ }^{[14]}$ Untreated GDM results in adverse maternal and fetal outcomes such as increased risk of preterm delivery, preeclampsia, macrosomia or large for gestational age (a known risk factor for birth injury), operative delivery, stillbirth, respiratory distress syndrome, and neonatal hypoglycaemia. ${ }^{[15]}$ The oral glucose tolerance test (OGTT) is considered to be the gold standard for diagnosis of GDM and is superior to fasting plasma glucose (FPG) and the glycosylated haemoglobin (HbAlc) level. ${ }^{[16]}$ However, the OGTT has its limitations including cost, the time required for the test and the unpleasant side-effects due to the high glucose load. Therefore, another screening method that can be performed during routine ultrasound assessment of pregnancy may be of value. ${ }^{[17]}$ Midtrimester fetal liver length (FLL) measurement is a potential test because of deposition of excess glucose in the form of glycogen in the fetal livers of mothers with diabetes. ${ }^{[18]}$

\section{Methods}

The study took place at the National Endocrine and Diabetic Centre, College of Medicine, Al-Mustansiriyha University in collaboration with the Department of Obstetrics and Gynecology, at Al-Yarmouk Teaching Hospital, Iraq. The study took place over a two-year period from September 2016 to September 2018. One hundred and twenty singleton pregnant women at high risk for GDM were recruited. FLL measurements were performed during routine ultrasound scan at 23 weeks followed by a $75 \mathrm{~g}$ OGTT at 24 weeks' gestation. Correlations between the mid-trimester FLL and OGTT were made. Gestational age was established using ultrasound fetal crown-rump length measurement at $10-12$ weeks' gestation. $\mathrm{HbA1c}$ was performed at booking as part of the screening tests for pre-existing diabetes. All participants gave verbal consent for their full medical and obstetric history including ultrasound examination and OGTT. Ethical approval was obtained from the Ethics Committee of the Obstetric and Gynecological Department.

Inclusion criteria included singleton pregnancy, normal random glucose levels (4.4 - $7.8 \mathrm{mmol} / \mathrm{L}), 22$ - 23 weeks' gestation, and risk factors for gestational diabetes. 
Women were ineligible for the study if they had multiple pregnancies, a congenital anomaly, were known diabetics or hypertensive patients and had any chronic disease that could affect fetal growth.

The FLL measurement was performed by ultrasound using a sagittal and coronal section of the fetal abdomen. The tip of the right lobe of the liver was clearly identified, and liver length measured from the dome of the left hemidiaphragm to the tip of the right lobe. The OGTT was performed after overnight fasting, with participants required to have had normal meals in the previous three days without deliberate dieting, and not to have smoked preceding the test. Samples were obtained from venous plasma to measure glucose levels at fasting and after 2 hours following the administration of $75 \mathrm{~g}$ glucose dissolved in $300 \mathrm{ml}$ water. According to the National Institute for Health and Care Excellence (NICE) guidelines, GDM is diagnosed when a pregnant woman has either: (i) a fasting plasma glucose level $\geq 5.6 \mathrm{mmol} / \mathrm{L}(100 \mathrm{mg} / \mathrm{dL})$ or (ii) a 2-hour plasma glucose level $\geq 7.8 \mathrm{mmol} / \mathrm{L}(140 \mathrm{mg} / \mathrm{dL})$.

\section{Statistical analysis}

Data analysis was performed using the Statistical Package for Social Sciences (IMB Corp., USA). Categorical data were presented as frequencies and percentages, and differences between proportions were determined by a $z$-test. Continuous variables were presented as means with standard deviations and differences between study groups were determined by student's $t$-test. Pearson's correlation was used to test the relation between FLL and maternal blood glucose levels at fasting, and at two hours following $75 \mathrm{~g}$ oral glucose load as part of the OGTT. A receiver operator curve used for determination of impaired glucose tolerance test results depending on the FLL (measured at 23 weeks' gestation by ultrasound). Binary logistic regression was performed to reveal the real size of differences and to account for confounders. Significance level was set at 0.05 .

\section{Results}

A total of 120 pregnant women at high risk for gestational diabetes aged 21 - 37 years, participated in the study. The incidence of gestational diabetes was $19.2 \%$.

As shown in Table 1, there were no differences in age and parity between the GDM v. non-GDM groups. Table 2 shows that the history of previous gestational diabetes was the most important risk factor for GDM, followed by a first-degree family history of diabetes.

Mean (SD) FLL values were significantly higher among GDM patients v. healthy pregnant females respectively: 37.2 (3.4) v. 33.1 (2.7); $p<0.001$. Univariate analysis showed that FLL in the GDM group was 1.6 times the non-GDM group (OR 1.6; 95\% CI 1.305 - 1.962) with a high specificity (95.9\%) and negative predictive value (95.9\%) for FLL for GDM in addition to high specificity and sensitivity. After adjustment for parity and cumulative risk factors, this finding did not change significantly (OR 1.599; 95\% CI 1.30 - 1.962).

\section{Discussion}

A number of studies have investigated the utility of FLL for the early prediction of gestational diabetes. ${ }^{[17-19]}$ Our study of Iraqi women at risk for GDM shows a high correlation between mid-trimester FLL measurement and OGTT blood glucose values. This is a significant finding as it offers an opportunity for early diagnosis of GDM, especially among pregnant women who cannot easily tolerate the OGTT.

Table 1. Comparison of the main characteristics of the study groups

\begin{tabular}{llll}
\hline Variable & GDM $(\mathbf{N = 2 3})$ & Non-GDM $(\mathbf{N = 9 7})$ & $p$-value \\
\hline Age (years), mean (SD) & $27.9(3.3)$ & $28.1(3.9)$ & 0.74 \\
Parity, $n$ (\%) & $2.0(1.3)$ & $1.7(1.2)$ & 0.45 \\
Parity groups, $n$ (\%) & & $19(19.6)$ & 0.81 \\
$\quad$ Nulliparous & $4(17.4)$ & $24(24.7)$ & 0.45 \\
$\quad$ Primipara & $4(17.4)$ & $25(25.8)$ & 0.65 \\
$\quad$ Secundigravida & $7(30.4)$ & $22(22.7)$ & 0.92 \\
$\quad$ Tercigravida & $5(21.7)$ & $7(7.2)$ & 0.36 \\
$\quad$ Multiparity $(\geq 4)$ & $3(13.0)$ & &
\end{tabular}

Table 2. Distribution of risk factors by study group

\begin{tabular}{|c|c|c|c|}
\hline Variable & GDM $(N=23), n(\%)$ & Non-GDM $(N=97) n(\%)$ & GDM + Non-GDM $(N=120), n(\%)$ \\
\hline \multicolumn{4}{|l|}{ Cumulative risk number } \\
\hline One & $20(87.0)$ & $90(92.8)$ & $110(91.7)$ \\
\hline Two & $3(13.0)$ & $7(7.2)$ & $10(8.3)$ \\
\hline \multicolumn{4}{|l|}{ Risk factors } \\
\hline First-degree family history of DM & $3(13.0)$ & $23(23.7)$ & $26(21.7)$ \\
\hline Age $>35$ years & 0 & $3(3.1)$ & $3(2.5)$ \\
\hline Glycosuria in 1st trimester & $1(4.3)$ & $7(7.2)$ & $8(6.7)$ \\
\hline Obese mother & 0 & $9(9.3)$ & $9(7.5)$ \\
\hline Previous congenital anomaly & 0 & $2(2.1)$ & $2(1.7)$ \\
\hline Previous GD & $12(52.2)$ & $47(48.5)$ & $59(49.2)$ \\
\hline Previous macrosomic baby & $7(30.4)$ & $5(5.2)$ & $12(10.0)$ \\
\hline Recurrent PE & 0 & $1(1.0)$ & $1(0.8)$ \\
\hline
\end{tabular}


Table 3. Validity values of fetal liver length in the determination of impaired glucose tolerance test results

\begin{tabular}{lll}
\hline & Validity values $\mathbf{( \% )}$ & $\mathbf{9 5 \%}$ CI \\
\hline Sensitivity & 82.6 & $60.5-94.3$ \\
Specificity & 95.9 & $89.2-98.7$ \\
PPV & 82.6 & $60.5-94.3$ \\
NPV & 95.9 & $89.2-98.7$ \\
AUC & 82.6 & $69.8-95.5$ \\
Accuracy & 93.3 & \\
CI = confidence interval; PPV = positive predictive value; NPV = negative predictive value; \\
AUC = area under the curve.
\end{tabular}

Our findings are supported in a study by Perovic et al. ${ }^{[18]}$ of 331 women pregnant with a single fetus and at high risk for gestational diabetes. In their study, a positive correlation was found between FLL measurements at the 23-week ultrasound and $100 \mathrm{~g}$ OGTT measured at 24 weeks' gestation. ${ }^{[18]}$ In another study, Roberts et al. ${ }^{[20]}$ measured FLL in 80 gestational diabetic women and the result of the measurements was significantly higher than expected at 18 weeks' gestation, also supporting the possibility of FLL in the early prediction of gestational diabetes. ${ }^{[20]}$ Mirghani et al. ${ }^{[21]}$ compared FLL measurements by ultrasound between 19 pregnant women with GDM and 104 women without GDM at $21-24$ weeks' gestation. FLL in GDM women exceeded that in pregnant women with a normal sugar profile as early as 21 weeks, in line with previous studies. ${ }^{[2]}$ Similarly, Elwahab et al. ${ }^{[22]}$ assessed the relation between mid-trimester ultrasound FLL at 20 - 24 weeks with a 75 g OGT in 150 singleton pregnant women with a high risk for GDM at $24-28$ weeks' gestation. The mean FLL in GDM was significantly greater than in normal pregnant women (36.55 v. $33.93 \mathrm{~mm}$, respectively; $p<0.001)$. The increment in liver size was, however, associated with maternal fasting glucose levels but not 1st- or 2nd-hour glucose level. ${ }^{[2]}$

In summary, these studies and our findings support the significance of FLL women as a correlate of GDM which we have demonstrated in Iraqi pregnant women at risk for GDM.

\section{Conclusion}

Measurement of FLL during routine mid-trimester ultrasound in women at high risk for GDM correlates highly with the OGTT taken later during pregnancy. Therefore, FLL represents an early important marker for the diagnosis of GDM.

Acknowledgements. None.

Author contributions. Equal contributions.
Funding. None.

Conflicts of interest. None.

1. American Diabetic Association. Classification and diagnosis of diabetes mellitus. Diab Care 2015; 38(Suppl 1):S8-S16. https://doi.org/10.2337/dc15-S005

2. Williams D. Medical complications of pregnancy. In: Kenny L, Myers J, eds. Obstetrics by Ten Teachers: Cleveland, 2017:306-309. https://doi.org/10.1201/9781315382401

3. Kampmann U, Madsen LR, Skajaa GO, Iversen DS, Moeller N, Ovesen P. Gestational diabetes: A clinical update. World J Diabetes 2015;6(8):1065-1072. https://doi.org/10.4239/wjd.v6.i8.1065

4. Mpondo BC, Ernest A, Dee HE. Gestational diabetes mellitus: Challenges in diagnosis and management. J Diabetes Metab Disord 2015;14(1):42. https://doi.org/10.1186/s40200-015-0169-7

5. Moore LE. Amount of polyhydramnios attributable to diabetes may be less than previously reported. World J Diabetes 2015;8(1):7-10. https://doi.org/10.4239/wjd.v8.i1.7

6. Farrar D. Hyperglycemia in pregnancy: Prevalence, impact, and management challenges. Int J Womens Health 2016;20(8):519-527. https://doi.org/10.2147/IJWH.S102117

7. Fahami F, Torabi S, Abdoli S. Prediction of glucose intolerance at $24-28$ weeks of gestation by glucose and insulin level measurements in the first trimester. Iran J Nurs Midwifery Res 2015;20(1):81-86.

8. Koning SH, Hoogenberg K, Lutgers HL, Van den Berg PP, Wolffenbuttel BH. Gestational diabetes mellitus: Current knowledge and unmet needs. J Diabetes 2016;8(6):770-781. https://doi. org/10.1111/1753-0407.12422

9. Usta A, Usta CS, Yildiz A, et al. Frequency of fetal macrosomia and the associated risk factors in pregnancies without gestational diabetes mellitus. Pan Afr Med J 2017;26:62. https://doig. org/10.11604/pamj.2017.26.62.11440

10. Mitanchez D, Yzydorczyk C, Simeoni U. What neonatal complications should the pediatrician be aware of in case of maternal gestational diabetes? World J Diabetes 2015;6(5):734-743. https:// doi.org/10.4239/wjd.v6.i5.734

11. Abu-Heija AT, Al-Bash MR, Al-Kalbani MA. Effects of maternal age, parity and pre-pregnancy body mass index on the glucose challenge test and gestational diabetes mellitus. J Taibah Univ Sci 2017;12(4):338-342. https://doi.org/10.1016/j.jtumed.2017.01.005

12. England L, Kotelchuck M, Wilson HG, et al. Estimating the recurrence rate of gestational diabetes mellitus (GDM) in Massachusetts 1998 - 2007: Methods and findings. Matern Child Health J 2015;19(10):2303-2313. https://doi.org/10.1007/s10995-015-1750-x

13. Moosazadeh M, Asemi Z, Lankarani KB, et al. Family history of diabetes and the risk of gestational diabetes mellitus in Iran: A systematic review and meta-analysis. Diabetes Metab Syndr: Clin Res Rev 2017;11(Suppl 1):S99-S104. https://doi.org/10.1016/j.dsx.2016.12.016

14. Huhn EA, Fischer T, Göbl CS, et al. Screening of gestational diabetes mellitus in early pregnancy by oral glucose tolerance test and glycosylated fibronectin: Study protocol for an international, prospective, multicentre cohort trial. BMJ Open 2016;6(10):e012115. https://doi.org/10.1136/ bmjopen-2016-012115

15. Sharma A, Agrawal A, Goel M, Gupta M. Utility of fasting plasma glucose test as screening tool for gestational diabetes mellitus based on International Association of the Diabetes and Pregnancy Study Group criteria. Int J Reprod Contracept Obstet Gynecol 2017;5(6):2005-2010. http://doi.org/10.18203/2320-1770.ijrcog20161707

16. Kim DL, Kim SD, Kim SK, Park S, Song KH. Is an oral glucose tolerance test still valid for diagnosing diabetes mellitus? Diabetes Metab J 2016;40(2):118-128. https://doi.org/10.4093/ dmj.2016.40.2.118

17. Gojnic M, Stefanovic T, Perovic M, et al. Prediction of fetal macrosomia with ultrasound parameters and maternal glycemic controls in gestational diabetes mellitus. Clin Exp Obstet Gynecol 2012;39(4):512-515.

18. Perovic M, Gojnic M, Arsic B, et al. Relationship between mid-trimester ultrasound fetal liver length measurements and gestational diabetes mellitus. J Diabetes 2015;7(4):497-505. https://doi.org/10.1111/1753-0407.12207

19. Fattah EA. Diagnostic Ability of the fetal ultrasonographic parameters in screening for gestational diabetes. MOJWH 2017;6(1):344-356. http:doi.org/10.1111/1753-0407.12207

20. Roberts AB, Mitchell J, Murphy C, Koya H, Cundy T. Fetal liver length in diabetic pregnancy. Am J Obstet Gynecol 1994;170(5):1308-1312. https://doi.org/10.1016/S0002-9378(13)90455-5

21. Mirghani H, Zayed R, Thomas L, Agarwal M. Gestational diabetes mellitus: Fetal liver length measurements between 21 and 24 weeks' gestation. J Clin Ultrasound 2007;35(1):34-37. https://doi.org/10.1002/jcu.20294

22. Elwahab AMA, Alkafrawy ME, Elghany AMA. The role of mid-trimester ultrasound fetal liver length measurement in prediction of gestational diabetes mellitus. Nat Sci 2018;16(5):90-94 https://doi.org/10.7537/marsnsj160518.12

Accepted 2 December 2019. 